\title{
Plasmodium berghei: Cerebral Malaria in CBA Mice Is Not Clearly Related to Plasma TNF Levels or Intensity of Histopathological Changes
}

\author{
L. J. M. Carvalho, H. L. Lenzi,* M. Pelajo-Machado,* D. N. Oliveira,* C. T. Daniel-Ribeiro, \\ and M. F. Ferreira-da-Cruz
}

Laboratory of Malaria Research, Department of Immunology and *Department of Pathology, Instituto Oswaldo Cruz, FIOCRUZ, 21045-900 Rio de Janeiro, RJ, Brazil

Carvalho, L. J. M., Lenzi, H. L., Pelajo-Machado, M., Oliveira, D. N., Daniel-Ribeiro, C. T., and Ferreira-da-Cruz, M. F. 2000. Plasmodium berghei: Cerebral malaria in CBA mice is not clearly related to plasma TNF levels or intensity of histopathological changes. Experimental Parasitology 95, 1-7. Plasmodium berghei ANKA infection in CBA/ $\mathrm{J}$ mice leads to the development of cerebral malaria $(\mathrm{CM})$ that kills $80-90 \%$ of the animals in 6-9 days. This model has been used to study the pathogenesis of CM, which is a major cause of morbidity and mortality in Plasmodium falciparum-infected individuals. The role of cytokines in the induction of CM in the murine model has been well documented, but most studies have been restricted to the peak of neurological manifestations. Here we used a sequential approach to compare mice that developed CM with those that developed no cerebral pathology. Animals were examined for systemic histopathological changes and plasma Tumor Necrosis Factor- $\alpha$ (TNF) levels. The objectives were (a) to further determine the importance of factors commonly associated with murine $\mathrm{CM}$ - such as elevated levels of TNF and the presence of hemorrhage and vascular plugging - by comparing mice at different stages of infection and/or with different outcomes following infection and (b) to examine the importance of systemic changescourse of parasitemia and histopathological alterations in brain, liver, and lungs - in the development of CM. The data suggest that (a) the clinical manifestation of CM appears to be associated with a wave of merozoite release on days 6-7, (b) murine CM does not present reliable histopathological indicators, (c) there is no topographic association between the occurrence of intravascular plugging and the hemorrhagic foci, (d) monocyte-monocyte and monocyte-endothelial cell adherence were the most expressive histopathological events and were not restricted to brain vessels, (e) blood levels of TNF are not indicative of the local tissue reaction, (f) adhesiveness of monocyte/endothelial cells fluctuate during infection and is dissociated from the lymphocyte homing to the liver, and (g) pulmonary megakaryocytosis (megakaryopoiesis?) is a late event in the lungs.

Index Descriptors and Abbreviations: cerebral malaria (CM); Plasmodium berghei ANKA; CBA/J mice; Tumor Necrosis Factor- $\alpha$ (TNF); cytokines; merozoite release; hemorrhages; histopathology; intercellular cell adhesion molecule-1 (ICAM-1); lymphocyte function antigen1 (LFA-1).

\section{INTRODUCTION}

Cerebral malaria (CM) is a frequently fatal complication of Plasmodium falciparum infection in man (WHO 1994). Other severe manifestations include pulmonary edema, renal failure, severe anemia, and hypoglycemia (Warrell et al. 1990). The murine model of CM, using the infection of CBA mice by $P$. berghei ANKA strain, has been widely used and shares some characteristics with human CM (Grau et al. 1989). Mice develop an acute infection that kills 80$90 \%$ of the animals in 6-9 days and that shows clinical and histopathological signs of neurologic involvement (Thumwood et al. 1988). The remaining 10-20\% die later (2 to 3 weeks after infection) by other malaria-related complications, such as severe anemia and hyperparasitemia (Grau et al. 1989). Murine CM has been shown to be due not to the infection itself, but rather a consequence of a strong immune reaction by the host and the release of cytokines (Clark and Rockett 1994; Clark et al. 1997; Finley et al. 1982; Grau et al. 1987, 1989). Many aspects of the pathogenesis of CM in this model have been elucidated; however, there are still no detailed descriptions of the kinetics of parasitemia and 
its association with development of CM nor has there been a sequential and integrated view of the infection and its pathology. We therefore performed a sequential study of the infection in this model by histopathological analysis of mice early in the infection (day 3), at the time of CM development (days 6-8, including mice with and without clinical CM), and after this critical period (mice that did not develop $\mathrm{CM}$, day 10). Analysis of mice 10 days postinfection (CM survivors) were used as reference for differences between this group and the mice that had developed CM. Observations arising from these approaches have provided new insights on the understanding of CM pathogenesis in this model and their relevance to the human situation.

\section{MATERIALS AND METHODS}

Animals, parasite, and infection. Four-to 8-week-old female CBA/ $\mathrm{J}$ mice were inoculated by intraperitoneal injection with $1 \times 10^{6}$ $P$. berghei ANKA-parasitized erythrocytes obtained from a donor infected CBA/J mouse. A total of 71 mice were infected and 17 used as controls, in three separate experiments.

Experimental design. A total of 32 infected mice were examined, on the following days after infection: 3 (10 mice), 6 ( 2 mice $), 7$ ( 8 mice), 8 ( 2 mice), and 10 (10 mice). Uninfected control mice (ageand sex-matched) were examined on days 3 (6 mice), 7 (6 mice), and 10 (5 mice). Blood samples were taken from 9 other infected, premoribund mice presenting clear signs of $\mathrm{CM}$ on day 6 , and plasma was assayed for TNF. Twenty-seven infected mice developed clinical signs of CM and died spontaneously ( 8 mice on day 5, 10 on day 6 , 6 on day 7, 3 on day 8). Three mice died on day 16 by malaria-related complications other than CM.

Parasitemia and clinical parameters. Density and maturation of circulating parasites were determined daily by examination of Giemsastained blood smears collected from the tail vein. Clinical CM was defined by the presentation of at least one of the following signs: limb paralysis, convulsions, stupor, and roll over. Spleen/body weight ratio was used to quantify the degree of splenomegaly. Hematocrit was measured in mice sacrificed on days 3, 7, and 10 .

Histopathology. Brain, lung, and liver samples were fixed in Carson's formalin-Millonig (Carson et al. 1993) and embedded in parafin. Sections $(5 \mu \mathrm{m})$ were stained with hematoxilin-eosin. For documentation, selected slides were analyzed in a confocal laser-scanning microscope (LSM 410, Zeiss), using He-Ne laser (633 nm) in transmittedlight channel with or without differential interference contrast (DIC). Images were transfered from LSM computer to Microsoft Imager, Corel Draw 6.0, and Powerpoint for final adjustments of contrast, brightness, and gamma correction, and then printed in a Codonics NP 1600 printer.

Quantitative analysis of cerebral hemorrhages was performed using Kontron Elektronik Imaging System KS 400, after manual demarcation of each individual hemorrhagic area. Intensity of vascular plugging and adherence of mononuclear cells in brain vessels was determined in a semiquantitative double-blinded reading of slides.
TNF detection. Plasma TNF levels were measured using the biological assay with WEHI 164 cells, as described by Espevik and NissenMeyer (1986) with minor modifications. Plasma samples were assayed in dilutions from 1:8 to 1:64. The concentration of the standard recombinant TNF- $\alpha$ giving the closest value corresponding to $50 \%$ lysis was determined as $8 \mathrm{pg} / \mathrm{ml}$ and used to calculate the TNF concentration in the test samples. By convention, test samples with values of lysis between 40 and $60 \%$ were taken as $8 \mathrm{pg} / \mathrm{ml}$ (and then multiplied by the reciprocal dilution). Values below $25 \%$ lysis were recorded as undetectable; values between 25 and $40 \%$ lysis were categorized as "lower than" $(<) 8 \mathrm{pg} / \mathrm{ml}$; and values over $60 \%$ lysis were assigned as "greater than" $(>) 8 \mathrm{pg} / \mathrm{ml}$. Levels of TNF were then classified as follows: undetectable (below $25 \%$ lysis), low ( $<64 \mathrm{pg} / \mathrm{ml}$ ), intermediate $(\geq 64<128 \mathrm{pg} / \mathrm{ml})$, or high $(\geq 128 \mathrm{pg} / \mathrm{ml})$.

Statistical analysis. Mann-Whitney and Kruskal-Wallis nonparametric tests were used to determine the statistical significance of the results obtained.

\section{RESULTS}

Characteristics of the infection. Inoculation of $P$. berghei ANKA to $\mathrm{CBA} / \mathrm{J}$ mice resulted in a lethal infection. Ten of 71 infected mice were examined on day 3 for early histopathological changes. Among the remaining 61 mice, 36 died spontaneously after displaying clinical signs of CM. Twelve mice were examined on days 6 ( 2 mice), 7 ( 8 mice), and 8 ( 2 mice); although half presented clinical signs of CM ( 2 mice in each day), all 12 mice had some evidence of brain lesions by histopathology. The 13 mice that remained did not develop clinical CM and were examined on day 10 (10 mice) or died spontaneously on day 16 (3 mice).

Parasitemia was detected in most of the mice starting on day 1 and reached values above $10 \%$ by days $6-7$. From day 7 to 10, parasitemia rose slightly. Although $P$. berghei is described as an asynchronic quotidian malaria parasite (Landau and Chabaud 1994), with a 22- to 25-h cycle, we noted a tendency toward synchronization of schizont rupture. Twice during the follow-up on days 3-4 and 6-7 a visible predominance of free merozoites and young trophozoites (ring forms) was observed; on the other days, a clear predominance of a parasite stage was not observed. It is important to point out that most of the infected mice died with clinical signs of CM on days 5-8, coincident with the second "wave of synchronic" merozoite release. From day 7 onward, young erythrocyte forms, such as normoblasts, appeared in the peripheral blood, and parasitized red blood cells were enlarged. In addition, infected mice had low hematocrits and splenomegaly, especially on day 10 (data not shown). The evolution of parasitemia during infection appears as follows: (a) day 0 to days 3-4, fast multiplication of parasites and 
the appearance of the first synchronic schizogony;(b) days 3-4 to days 6-7, progressive increase in parasitemia and the appearance of a second wave of merozoite release, with development of CM; and (c) days 7 to 10 , a relatively stable parasitemia in surviving mice with development of anemia (low hematocrit and presence of normoblasts in peripheral blood).

TNF levels. Plasma TNF levels were determined in 15 noninfected control and 34 infected mice. None of the control mice or infected mice examined on day 3 had detectable levels of TNF. The 15 mice with clinical signs of CM that were assayed for TNF (11 mice on day $6 ; 2$ on day 7; and 2 on day 8 ) had intermediate (6 mice) to high (9 mice) levels. In the 6 mice without clinical signs of CM that were assayed on day 7 , the TNF levels varied from undetectable (1 mouse) to low (1 mouse), intermediate (3 mice), or high (1 mouse) levels. Mice examined on day 10 had undetectable (4 mice), low (2 mice), or intermediate (2 mice) levels.

Histopathology of the central nervous system. Although only 6 had clinical signs of CM, all 12 mice examined on days 6-8 had histopathological changes in cerebrum and cerebellum. The mean size of individual hemorrhagic areas ranged from 1210 to $9033 \mu \mathrm{m}^{2}$ (total mean $=2670 \pm 2205$ $\mu \mathrm{m}^{2}$ ). When comparing mice with or without clinical signs of CM, data were as follows in each group: (a) hemorrhagic areas measured $3922 \pm 3061$ and $1776 \pm 658 \mu \mathrm{m}^{2}$, affecting from 0.06 to $0.93 \%$ (median: $0.19 \%$ ) and from 0.02 to $0.25 \%$ (median: $0.04 \%$ ) of the total cerebral area; (b) areas larger than $2000 \mu \mathrm{m}^{2}$ accounted for $23-61 \%$ (median: $40 \%$ ) and from 19 to $55 \%$ (median: $28 \%$ ) of all hemorrhages; (c) number of hemorrhages $/ \mathrm{mm}^{2}$ ranged from 0.33 to 2.18 (median: 0.65 ) and from 0.13 to 2.05 (median: 0.16 ), in mice with or without $\mathrm{CM}$, respectively. The number and size of hemorrhagic areas did not correlate with the TNF levels, although there was a tendency for such a correlation by grouping individual data, and the mouse which presented the largest hemorrhagic area showed the highest level of TNF. Qualitative histopathological findings included (a) several perivascular or laminated microhemorrhagic areas mainly in cerebellum and in olfactory brain (Fig. 1 (1)), (b) subarachnoid hemorrhages, and (c) perivascular edema with few extravasated mononuclear cells. In addition, in some mice, damage of Purkinje cells and hippocampal neurons, vascular plugging by pigment-containing monocytes, and monocyte adherence to endothelial cells (Fig. 1 (2)), with frequent intravascular mitotic figures and sometimes with small and activated lymphocytes were observed. Vascular plugging and adherence of monocytes to endothelial cells were intense and similar in all animals on days 6-8. Hemorrhages and vascular plugging were topography independent - that is, hemorrhages were not necessarily adjacent to congested vessels. None of the infected mice studied on day 3 , and only 1 of 10 animals analyzed on day 10, had brain hemorrhages. This single affected mouse had a delayed parasitemia and the highest TNF levels compared to other infected mice assayed on day 10 . The remaining 9 mice examined on day 10 had monocyte-monocyte adherence that was more persistent than monocyte-endothelial cell adherence and had some but much lower intravascular congestion than in those observed on days 6-8.

Histopathology of the liver. Malarial pigment in Kupffer cells was detectable on day 3 as was an incipient mononuclear cell adhesion to the vascular endothelium in some animals. On days 6-8, the adhesion of pigment-containing monocytes to themselves, to the endothelium, and among themselves was remarkably intensified, forming true intravascular networks (Fig. 1(3)). Kupffer cells were hypertrophic and heavily pigmented; lymphocytes, pigment-containing monocytes, and mainly erythroid cells were present in enlarged sinusoids, forming cell clusters (Fig. 1(4)). Two of 12 mice had periductal portal infiltrates by mononuclear cells. The cytoplasm of many hepatocytes was microvacuolated. On day 10, infected mice showed triaditis by small, medium, and large lymphocytes, with occasional formation of lymphoid nodules without germinal centers and clusters of plasmacytes (Fig. 1 (5)). Intrasinusoidal lymphoid clusters were often observed, sometimes with activated lymphocytes. Sinusoids and portal lymphatic vessels were enlarged. Pigment-containing Kupffer cells were more hypertrophic than on days 6-8, and the pigment granulation was rougher. Monocyte adhesion was much less intense in all but one animal, which also presented cerebral lesions. Megakaryocytes were occasionally found in sinusoids.

Histopathology of the lungs. Lungs collected on day 3 from uninfected and infected mice were similar. By days 6-8, lungs from infected mice had intense adhesiveness of pigment-containing monocytes and, to a lesser degree, of neutrophils, to the endothelium. Ten of 12 infected mice showed intraalveolar macrophages and septal pneumonitis characterized by monocyte infiltrates, giving a compact aspect to lungs. One animal had a diffuse pulmonary edema. On day 10, most infected animals had pulmonary changes similar to those observed on days $6-8$, but leucocyteendothelial cell adhesiveness was less intense and all mice had several megakaryocytes within capillary vessels (Fig. 1(panel 6)). Two of 10 mice showed intraalveolar edema. 

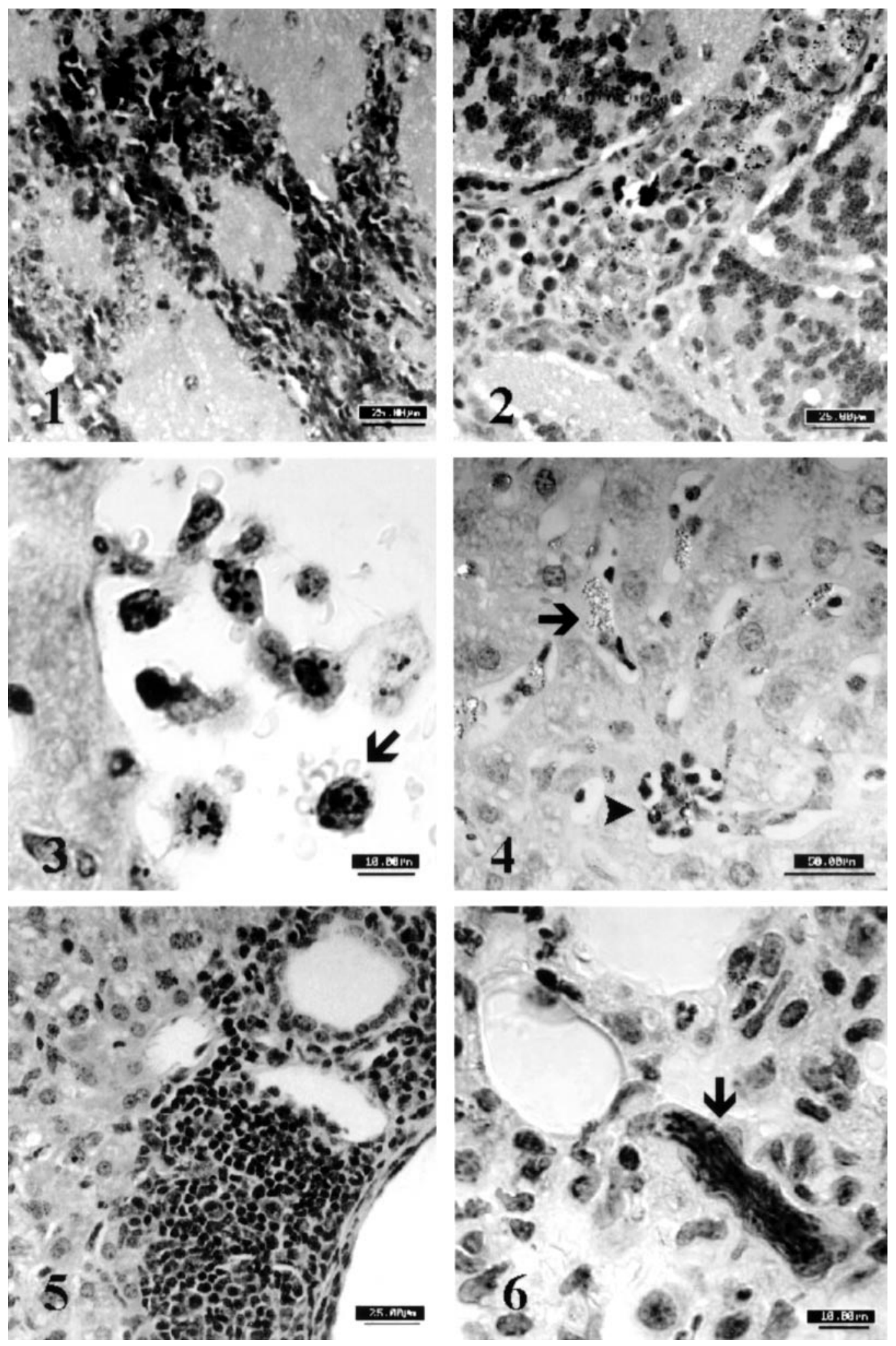


\section{DISCUSSION}

Cerebral malaria in humans and mice differ in many aspects. In mice, vascular plugging by monocytes and high levels of TNF have been associated with CM. In humans, postmortem histopathological findings reveal that parasitized erythrocytes rather than monocytes are sequestered in brain capillary vessels (Berendt et al. 1994); in addition, in vitro data such as rosetting properties of some parasite isolates (Chen et al. 1998) have been correlated with human $\mathrm{CM}$. A role for TNF in human CM pathogenesis remains undetermined. Some studies suggest that patients with $\mathrm{CM}$ have higher levels of circulating TNF and TNF receptors (Grau et al. 1992; Kern et al. 1992), while others do not show such a correlation (Yamada-Tanaka et al. 1996; Turner et al. 1998). However, studies of the pathogenic mechanisms and factors related to human CM are hampered by obvious limitations, and the mouse model remains one of the few alternatives to study the pathogenesis of CM.

We have shown in this study that development of CM in P. berghei ANKA-infected CBA/J mice was associated with a wave of merozoite release on days 6-7. Activation of the immune system, mainly $\mathrm{T}$ cells, occurs early in the infection and, on days $6-8$, there is a strong involvement of lymphoid organs, such as lymph nodes, spleen white pulp, and Peyer's patches, and of cells of lymphoid and myeloid origin (data not shown). These observations suggest that cytokines may have been released from the beginning of the infection and led to a striking activation of the immune system. Even microglial cell activation has been observed early in infection (Medana et al. 1996).

In this study mice were analyzed in different and sequential stages of infection, correlating quantitatively the histopathological changes, mainly hemorrhagic foci, with the clinical status and plasma TNF levels. When comparing mice killed on days $6-8$, we detected the following tendencies in those presenting clinical CM: (a) higher levels of TNF and (b) hemorrhagic foci that were more frequent and larger and occupied a higher percentage of the total cerebral area. However, the results showed that, due to individual variation, none of the parameters analyzed were absolute indicators of CM. Although administration of anti-TNF antibodies prevent murine CM (Grau et al. 1987), the demonstration in this study that plasma levels of TNF do not show a correlation with either clinical signs or histopathological changes at the individual level in this same model supports the interpretation that local levels of this cytokine, associated with changes such as vascular congestion, would be more important in determining the outcome of infection than systemic levels (Jennings et al. 1997).

The absence of hemorrhages in organs other than the brain and the topographic dissociation of congested vessels and hemorrhages in the central nervous system suggest that the occurrence of hemorrhages is not due to a damage following excessive pressure or other action of the plugging on the vessel walls, but could result from microendothelial damage selectively affecting brain vessels. The mild changes in the brains of infected mice that survived to day 10 remain undetermined, leaving the following two obvious hypotheses: (1) they developed acute changes and then, for unknown reasons, were able to recover or (2) for unknown reasons these mice were protected from cerebral disease and never developed such alterations.

Although infected mice that survived to day 10 had an absence of brain lesions, they did have an accumulation of mononuclear cells in the liver and a much smaller degree of monocyte adhesion to the endothelium, as well as a more organized reaction in secondary lymphoid organs, including lymph nodes, spleen white pulp, and Peyer's patches (data not shown). These characteristics might be related to the differential expression of adhesion as well as other regulatory molecules, whose modulation could have profound effects on the outcome of the infection. The intense adhesiveness of leucocytes, notably monocytes, to the endothelium of microvessels and among themselves was particularly striking in the liver and lungs of animals examined on days 6-8. In the liver, adhesion was particularly intense and reflected the strong activation of monocytes and endothelial cells, the former acquiring the aspect of macrophages and containing large quantities of malarial pigment. In peripheral blood, we have found only conventional monocytes without malarial

FIG. 1. (1) Microhemorrhagic areas in olfatory brain (8 days after infection). (2) Cerebellar vessel full of pigment-containing monocytes (7 days after infection). (3) Pronounced monocyte adherence on hepatic portal venule endothelium, forming an intravasular connecting mesh with long cytoplasmic extensions. Monocytes look like macrophages, contain malarial pigment, and eventually present adhered red blood cells on the surface (arrow) (7 days after infection). (4) Liver with hypertrophic Kupffer cells containing malarial pigment (arrow) and small intrasinusoidal aggregate of erythroid cells centered by a pigment-containing monocyte (arrowhead) (7 days after infection). (5) Portal mononuclear cell infiltrate forming lymphocytic pseudonodule (10 days after infection). (6) Elongated megakaryocyte (arrow) in lung capillary vessel (10 days after infection). 
pigment, suggesting that activated monocytes-macrophages did not circulate.

In addition to degenerative changes, liver histopathology revealed two other prominent changes: (a) intense adhesion phenomena, especially on days 6-8 (above), and (b) a tendency during infection to increase homing characteristics by lymphocytes and erythroid cells to accumulate in sinusoids and portal space, especially on day 10. Migration of lymphocytes to the liver, forming intrasinusoidal clusters and portal infiltrates, could lead to a more effective antimalarial immune response, as suggested by Playfair and de Souza (1982). There is also the possibility that these clusters reflect only a chronologic phenomenon rather than a real difference between the groups of mice killed on days 6-8 and on day 10. The time-space dissociation of these two phenomenaadhesion and lymphocyte migration-strengthen the concept that complex mechanisms of modulation of cell adhesion molecules are taking place during P. berghei ANKA infection.

In summary, our data suggest that during a $P$. berghei ANKA infection in CBA/J mice (a) clinical manifestations of $\mathrm{CM}$ are associated with a wave of merozoite release on days 6-7, (b) murine CM does not present secure histopathological indicators, (c) there is no topographic association between the occurrence of intravascular plugging and the hemorrhagic foci, (d) monocyte-monocyte and monocyteendothelial cell adherence were the most expressive histopathological events and were not restricted to brain vessels, (e) circulating levels of TNF might not reflect what is going on in the intimacy of the local tissue reaction, (f) adhesiveness of monocyte/endothelial cells is up- and down-regulated during the infection and is dissociated from the lymphocyte homing to the liver, and (g) pulmonary megakaryocytosis (megakaryopoiesis?) was a late event in the lungs.

\section{ACKNOWLEDGMENTS}

The authors dedicate this paper in honor of the Instituto Oswaldo Cruz on the occasion of the Centenary of its foundation, May 25th, 1900. The authors are grateful to Bruno Silva Vale and Arturo Max Arana-Pino for processing images in scanning light confocal microscopy, to Isaac Lima da Silva Filho and Janete Cuba from Evandro Chagas Hospital-IOC-Fiocruz for hematological analysis, to Belmira Ferreira dos Santos from the Fiocruz Central Animal House for providing us with CBA/J mouse strain and to Dr. Heitor Franco Jr. (IMT-São Paulo, Brazil) for kindly providing the P. berghei ANKA strain. This work was supported by funds of the Conselho Nacional de Desenvolvimento Tecnológico $(\mathrm{CNPq})$ and FAPERJ-Brazil.

\section{REFERENCES}

Berendt, A. R., Turner, G. D. H., and Newbold, C. I. 1994. Cerebral malaria: The sequestration hypothesis. Parasitology Today 10, $412-414$

Carson, F. L., Martin, J. H., and Lynn, J. A. 1993. Formalin fixation for electron microscopy: A re-evaluation. American Journal of Clinical Pathology 59, 365-373.

Chen, Q., Barragan, A., Fernandez, V., Sundström, A., Schlichtherle, M., Sahlén, A., Carlson, J., Datta, S., and Wahlgren, M. 1998. Identification of Plasmodium falciparum erythrocyte membrane protein 1 (PfEMP1) as the rosetting ligand of the malaria parasite P. falciparum. Journal of Experimental Medicine 187, 15-23.

Clark, I. A., and Rockett, K. A. 1994. The cytokine theory of human cerebral malaria. Parasitology Today 10, 410-412.

Clark, I. A., Al Yaman, F. M., and Jacobson, L. S. 1997. The biological basis of malarial disease. International Journal of Parasitology 27, 1237-1249.

Espevik, T., and Nissen-Meyer, J. 1986. A highly sensitive cell line, WEHI 164 clone 13, for measuring cytotoxic factor/tumor necrosis factor from human monocytes. Journal of Immunological Methods 95, 99-105.

Finley, R. W., Mackey, L. J., and Lambert, P-H. 1982. Virulent P. berghei malaria: Prolonged survival and decreased cerebral pathology in cell-deficient nude mice. Journal of Immunology 129, 2213-2218

Grau, G. E., Fajardo, L. F., Piguet, P. F., Allet, B., Lambert, P. H., and Vassalli, P. 1987. Tumor necrosis factor (cachectin) as an essential mediator in murine cerebral malaria. Science 237, 1210-1212.

Grau, G. E., Piguet, P. F., Vassalli, P., and Lambert, P. H. 1989. Tumor necrosis factor and others cytokines in cerebral malaria: Experimental and clinical data. Immunology Reviews 112, 49-70.

Jennings, V. M., Actor, J. K., Lal, A. A., and Hunter, R. L. 1997. Cytokine profile suggesting that murine cerebral malaria is an encephalitis. Infection and Immunity 65, 4883-4887.

Kern, P., Hemmer, C. J., Gallati, H., Neifer, S., Kremsner, P., Dietrich, H., and Porzsolt, F. 1992. Soluble tumor necrosis factor correlates with parasitemia and disease severity in human malaria. Journal of Infectious Diseases 166, 930-934.

Landau, I., and Chabaud A. 1994. Plasmodium species infecting Thamnomys rutilans: A zoological study. Advances in Parasitology 33, 49-90.

Medana, I. M., Hunt, N. H., and Chan-Ling, T. 1996. Early activation of microglia in the pathogenesis of fatal murine cerebral malaria. Glia 19, 91-103.

Playfair, J. H., and De Souza, J. B. 1982. Lymphocyte traffic and lymphocyte destruction in murine malaria. Immunology 46, 125-133.

Thumwood, C. M., Hunt, N. H., Clark, I. A., and Cowden, W. B. 1988. Breakdown of the blood-brain barrier in murine cerebral malaria. Parasitology 96, 579-589. 
Turner, G. D. H., Chuong, L. V., Mai, N. T. H., Chau, T. T. H., Phu, N. H., Bethell, D., Wyllie, S., Louwrier, K., Fox, S. B., Gatter, K. C., Day, N. P., Hien, T. T., White, N. J., and Berendt, A. R., 1998. Systemic endothelial activation occurs in both mild and severe malaria. American Journal of Pathology 152, 1477-1487.

Warrell, D. A., Molyneux, M. E., and Beasles, P. F. 1990. Severe and complicated malaria. Transactions of the Royal Society of Tropical Medicine and Hygiene 84, 1-65.
WHO, 1994. Weekly Epidemiological Record 69, 309-316.

Yamada-Tanaka, M. S., Ferreira-da-Cruz, M. F., Alecrim, M. G., Mascarenhas, L. A., and Daniel-Ribeiro, C. T. 1996. Tumor necrosis factor alpha, interferon gamma and macrophage stimulating factor in relation to the severity of Plasmodium falciparum malaria in the Brazilian Amazon. Tropical Geography and Medicine 47, 282-285.

Received 12 October 1998; accepted with revision 21 March 2000 